\title{
A NOTE ON TWO RECENT RESULTS ABOUT POLYNOMIALS WITH RESTRICTED ZEROS
}

\author{
ABDUllah Mir AND AJAZ WANI
}

\begin{abstract}
Let $P(z)$ be a polynomial of degree $n$ and for any complex number $\alpha$, let $D_{\alpha} P(z):=$ $n P(z)+(\alpha-z) P^{\prime}(z)$ denote the polar derivative of the polynomial $P(z)$ with respect to $\alpha$. In this paper, we first extend a recently proved result contained in a paper published in this journal to the polar derivative of a polynomial. We shall also point out a fault in other result published in the same paper and discuss in detail the validity of that result.
\end{abstract}

Mathematics subject classification (2010): 30A10, 30C10, 30C15.

Keywords and phrases: Bernstein inequality, polar derivative, polynomials.

\section{REFERENCES}

[1] A. AziZ And W. M. Shah, Some integral mean estimates for polynomials, Anal. Theory and Appl., 23 (2007), 101-111.

[2] T. N. Chan And M. A. Malik, On Erdös-Lax theorem, Proc. Indian Acad. Sci.(Math. sci.), 92 (1983), 191-193.

[3] K. K. DEWAN, N. Singh AND A. MiR, Extensions of some polynomial inequalities to the polar derivative, J. Math. Anal. Appl., 352 (2009), 807-815.

[4] K. K. Dewan AND S. Hans, On extremal properties for the derivative of polynomials, Math. Balkanica, 23 (2009) 27-35.

[5] P. D. LAX, Proof of a conjecture of P. Erdös on the derivative of a polynomial, Bull. Amer. Math. Soc., 50 (1944), 509-513.

[6] S. Kumar And R. LAL, Generalizations of some polynomial inequalities, Int. Elec. J. Pure Appl. Math., 3 (2011), 111-117.

[7] M. A. MaLiK, On the derivative of a polynomial, J. London Math. Soc., 1 (1969), 57-60.

[8] K. M. NAKPRASIT AND J. SOMSUWAN, An upper bound of a derivative for some class of polynomials, J. Math. Inequal., 11 (2017), 143-150.

[9] M. S. PUKHTA, Extremal problems for polynomials and on location of zeros of polynomials, Ph.D. Thesis, Jamia Millia Islamia, New Delhi, 1995. 